\title{
Optimal ITO transparent conductive layers for InGaN-based amber/red light-emitting diodes
}

\author{
Zhe Zhuang, D Daisuke IIda, Pavel Kirilenko, Martin \\ VELAZQUEZ-RIZO, D AND KAZUHIRO OHKAWA* \\ Computer, Electrical and Mathematical Sciences and Engineering (CEMSE) Division, King Abdullah \\ University of Science and Technology (KAUST), Thuwal, 23955-6900, Saudi Arabia \\ *kazuhiro.ohkawa@kaust.edu.sa
}

\begin{abstract}
Fabrication of indium tin oxide (ITO) was optimized for InGaN-based amber/red light-emitting diodes (LEDs). A radiofrequency sputtering reduced the sheet resistivity of ITO at low pressures, and a subsequent two-step annealing resulted in a low sheet resistivity (below $2 \times 10^{-4} \Omega \mathrm{cm}$ ) and high transmittance (over 98\%) in the amber and red regions between $590 \mathrm{~nm}$ to $780 \mathrm{~nm}$. Double ITO layers by sputtering could form an excellent ohmic contact with p-GaN. Application of the double ITO layers on amber and red LEDs enhanced light output power by $15.6 \%$ and $13.0 \%$, respectively, compared to those using ITO by e-beam evaporation.
\end{abstract}

(C) 2020 Optical Society of America under the terms of the OSA Open Access Publishing Agreement

\section{Introduction}

During the past decade there has been a tremendous development of III-nitride semiconductors and their visible-UV optoelectronic devices, especially efficient blue light-emitting diodes (LEDs) for bright and energy-saving white light sources [1-5]. Nowadays, InGaN-based blue LEDs have been successfully commercialized and achieve external quantum efficiencies (EQE) as high as $84.3 \%$ [6].

However, the efficiency of InGaN-based LEDs is still low in the amber and red regions due to the strong quantum-confined Stark effect (QCSE) and high defect density in high-In-content InGaN quantum wells (QWs) [7,8]. Red emission can be achieved with high efficiency using phosphide-based (Al, Ga, In)P semiconductor materials, although this is sensitive to ambient temperatures $[9,10]$. The efficiency of AlInGaP-based LEDs rapidly decreases with temperature, making it unsuitable for high temperature operations. Conversely, the efficiency of InGaN-based LEDs is expected to have little temperature dependence due to its large band-offsets in conduction and valence bands. InGaN-based LEDs have the potential for high-power applications at high temperatures.

We developed a novel high-temperature growth technique to grow high-quality and highIn-content InGaN layers, and demonstrated a long-wavelength electroluminescence (EL) peak emission of $740 \mathrm{~nm}[11,12]$. Additionally, we adopted strain-compensation methods by introducing AlN/AlGaN barriers [13] and hybrid QWs [14,15] to reduce defects and enhance light output power. Also, we have demonstrated 665-nm InGaN-based red LEDs on $\beta-\mathrm{Ga}_{2} \mathrm{O}_{3}$ substrates, allowing very low forward voltages below $2.5 \mathrm{~V}$ at $20 \mathrm{~mA}$ [16]. Other groups have also reported several approaches to obtain high-In-content InGaN-based LEDs, including InGaN/GaN multiple quantum dots [17], semipolar InGaN buffer layers [18], InGaN/GaN nanowires [19], and $\mathrm{InGaN} / \mathrm{GaN}$ QWs on lattice-matched $\mathrm{InGaN} / \mathrm{ScAlMgO}_{4}(0001)$ templates [20]. Additionally, a unique nitride-based red LED has also been reported, which utilizes Eu-doped GaN instead of In $\mathrm{GaN}$ as the active layer [21].

In addition to the crystal quality of active layers, high-quality $\mathrm{p}-\mathrm{GaN}$ contacts remain a challenge due to the low doping level and high work function of the p-GaN layer [22,23]. Different optical characteristics of $\mathrm{p}-\mathrm{GaN}$ contacts are required for different kinds of LEDs. For example, the flip-chip and vertical structure LEDs require a high level of reflectivity of p-GaN 
contacts [24], while the standard LEDs emitted from the top side require highly transparent p-contacts [25]. Indium tin oxide (ITO) has been widely used for the transparent p-electrodes in InGaN-based LEDs [26]. By optimizing the thermal annealing, ITO deposited at $300^{\circ} \mathrm{C}$ by e-beam evaporation could realize a high transmittance of above $96 \%$ in the visible region [27]. Also, ITO could be deposited by magnetron sputtering and obtained the transmittance above $90 \%$ with optimized oxygen flow rate [28,29]. However, these deposition processes usually introduce oxygen gas and adopt high-temperature conditions, complicating the process and requiring additional configurations for facilities. Furthermore, little attention has been paid to the optimization of ITO for InGaN-based LEDs in the amber and red regions. Although ITO is used for phosphide red LEDs, its transmittance has been limited to $90 \%$, thus far [30]. ITO/Al-doped zinc oxide (AZO) composite films were proposed to improve the transmittance to more than $96 \%$ [31], but they reduced reproducibility and productivity since AZO films require an additional deposition process in other facilities.

In this work, we systematically investigated the performance of ITO deposited by RF sputtering or e-beam evaporation. Both sheet resistivity and transmittance were optimized for InGaN-based amber and red LEDs. We propose a two-step annealing process under $\mathrm{O}_{2}$-containing and $\mathrm{O}_{2}$-poor atmospheres (in sequence), achieving a low sheet resistivity below $2 \times 10^{-4} \Omega \mathrm{cm}$ and high transmittance of more than $98 \%$ in the $590 \mathrm{~nm}$ to $780 \mathrm{~nm}$ wavelength range. The wavelength range covers the whole of the amber and red regions. For the contacts with p-GaN layers, double ITO layers were utilized in both amber and red LEDs, which achieved a lower forward voltage and higher light output power compared to an ITO layer by e-beam evaporation.

\section{Experimental details}

The InGaN-based amber and red LED epitaxial wafers were grown on $c$-plane patterned sapphire substrates by metalorganic vapor phase epitaxy (MOVPE) in a single-wafer horizontal reactor at $100 \mathrm{kPa}$ [11,12]. Trimethylgallium (TMGa), trimethylaluminum (TMAl), trimethylindium (TMIn), and $\mathrm{NH}_{3}$ were used as $\mathrm{Ga}, \mathrm{Al}, \mathrm{In}$, and $\mathrm{N}$ sources, respectively. The n-type and p-type dopant sources of $\mathrm{Si}$ and $\mathrm{Mg}$ were $\mathrm{N}_{2}$-diluted silane and biscyclopentadienylmagnesium, respectively. During MOVPE growth, carrier gases were $\mathrm{N}_{2}, \mathrm{H}_{2}$, or a mixture of the two. The schematic epitaxial structures of the InGaN-based amber and red LEDs have been reported in previous studies by our laboratory $[14,15]$.

The ITO was prepared on double-sided polished sapphire substrates (DPSS) or GaN on DPSS through e-beam evaporation or RF sputtering at room temperature (RT) without oxygen gas. The ITO target was comprised of $90 \mathrm{wt}$. \% $\mathrm{In}_{2} \mathrm{O}_{3}$ and $10 \mathrm{wt} . \% \mathrm{SnO}_{2}$. Since this approach is quite simple and does not require additional heaters or gases, it can be applied to most of the sputtering or e-beam evaporation systems. In the case of RF sputtering, different sputtering pressures were used to investigate the electrical and optical performance of deposited ITO layers. A subsequent two-step thermal annealing was proposed and optimized under different conditions. Finally, optimal ITO layers were used as transparent conductive layers for amber and red LEDs. The ITO layers were first deposited on the $\mathrm{p}-\mathrm{GaN}$ layer by RF sputtering or e-beam evaporation. Then, we formed a mesa structure of the ITO layers by inductively coupled plasma etching, and deposited $\mathrm{Cr} / \mathrm{Ni} / \mathrm{Au}(50 \mathrm{~nm} / 20 \mathrm{~nm} / 200 \mathrm{~nm})$ as the electrode pads on the ITO and the n-type layer. The typical size of the LEDs was $400 \times 400 \mu \mathrm{m}^{2}$.

A standard four-point probe technique was used to measure the sheet resistivity of ITO. A UV-vis spectrophotometer evaluated the transmittance of each ITO layer. For ITO on DPSS, the transmittance was normalized using the DPSS, while for ITO on GaN on DPSS, the transmittance was normalized using GaN on DPSS. The sheet resistivity and transmittance of ITO were regarded as the critical parameters for optimization. Because this work was focused on the amber and red emission from LEDs, we paid more attention to the transmittance from $590 \mathrm{~nm}$ to $780 \mathrm{~nm}$. The structures of as-deposited and annealed ITO were analyzed by x-ray diffraction 
(XRD) using $\mathrm{Cu}(\mathrm{K} \alpha)$ radiation $(\lambda=1.5406 \AA)$. The chemical states of ITO annealed in different atmospheres were analyzed by X-ray photoelectron spectroscopy (XPS). Electrical pumping of all LED devices was implemented in the probe station at RT.

\section{Results and discussion}

Figures 1(a) and 1(b) show the sheet resistivity and transmittance of as-deposited ITO with a thickness of around $75 \mathrm{~nm}$ on DPSS, respectively. These ITO layers were deposited by RF sputtering or e-beam evaporation, respectively. The deposited pressure of RF sputtering was investigated from 3 mTorr to 10 mTorr. It was found that the deposited pressure has a significant influence on the sheet resistivity of as-deposited ITO by RF sputtering. With the pressure decreasing from 10 mTorr to 3 mTorr, the sheet resistivity of as-deposited ITO by RF sputtering was reduced from $5.6 \times 10^{-2} \Omega \mathrm{cm}$ to $8.9 \times 10^{-4} \Omega \mathrm{cm}$, nearly two orders of magnitude. This phenomenon can be explained in terms of the energy of sputtered atoms onto the substrate [32]. At the lower pressure, fewer sputtered atoms collided with Ar atoms and thus had sufficient energy to form larger grains when arriving at the surface of the substrate. The larger grains resulted in reduced grain boundary scattering, leading to lower sheet resistivity of the films.
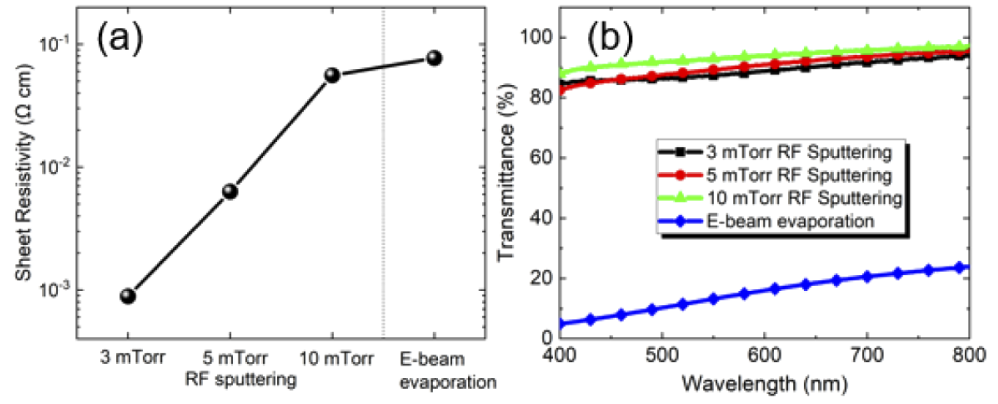

Fig. 1. (a) The sheet resistivity and (b) transmittance of as-deposited ITO layers by e-beam evaporation and RF sputtering with different deposited pressures.

However, the transmittance of as-deposited sputtered ITO slightly decreased in the wavelength range above $590 \mathrm{~nm}$ when the sputtering pressure was decreased from 10 mTorr to 3 mTorr. The maximum reduction of transmittance was 5 percentage points (from 93\% to 88\%) at the wavelength of $590 \mathrm{~nm}$. This reduction was attributed to the smoother surface of ITO deposited at lower pressure, which decreased the light extraction from the interface of ITO and air and led to the reduction of the transmittance [33]. In summary, the low-pressure sputtering can decrease the sheet resistivity by orders of magnitude, but only reduces the transmittance by a few percentage points. Therefore, in the case of sputtered ITO, the low pressure of 3 mTorr was adopted for the subsequent optimizing processes.

The performance of as-deposited ITO by e-beam evaporation was also plotted in Fig. 1 for comparison purposes. The sheet resistivity and transmittance of as-deposited ITO by ebeam evaporation were around $7.7 \times 10^{-2} \Omega \mathrm{cm}$ and less than $25 \%$, respectively. These worse performances compared to sputtered ITO can be attributed to a worsened crystal quality during the e-beam evaporation process [33].

Because as-deposited ITO by sputtering or e-beam evaporation had poor crystallinity, it was essential to use a subsequent annealing process to form stable polycrystalline films and improve the electrical and optical qualities [34]. In this work, two annealing processes in an oxygen-containing $\left(\mathrm{Ar} / \mathrm{O}_{2}\right.$ mixture) and oxygen-poor $(\mathrm{Ar})$ atmosphere were carried out at $600^{\circ} \mathrm{C}$. The annealing conditions for ITO by RF sputtering and e-beam evaporation are listed in Tables 1 and 2, respectively. The ITO layers with a thickness of $148 \mathrm{~nm}$ by RF sputtering were named 
S1-S3, while those with a thickness of $114 \mathrm{~nm}$ by e-beam evaporation were named E1-E7. Samples were prepared by RF sputtering or e-beam evaporation together, at the same time, to minimize variation in each fabrication.

Table 1. Annealing Conditions for Samples S1-S3 by RF Sputtering.

\begin{tabular}{cccccc}
\hline \multirow{2}{*}{ Sample } & \multicolumn{2}{c}{1 st annealing } & & \multicolumn{2}{c}{ 2nd annealing } \\
\cline { 2 - 3 } \cline { 5 - 6 } & Gas/flow rate $[\mathrm{sccm}]$ & Time $[\mathrm{min}]$ & & Gas/flow rate $[\mathrm{sccm}]$ & Time $[\mathrm{min}]$ \\
\hline $\mathrm{S} 1$ & $\mathrm{Ar} / 500$ & 5 & & $\mathrm{Ar} / 400 \mathrm{O}_{2} / 100$ & 1 \\
$\mathrm{~S} 2$ & $\mathrm{Ar} / 400 \mathrm{O}_{2} / 100$ & 1 & & $\mathrm{Ar} / 500$ & 5 \\
$\mathrm{~S} 3$ & $\mathrm{Ar} / 400 \mathrm{O}_{2} / 100$ & 5 & $\mathrm{Ar} / 500$ & 5 \\
\hline
\end{tabular}

Table 2. Annealing Conditions for Sample E1-E7 by E-beam Evaporation.

\begin{tabular}{cccccc}
\hline \multirow{2}{*}{ Sample } & \multicolumn{2}{c}{ 1st annealing } & & \multicolumn{2}{c}{ 2nd annealing } \\
\cline { 2 - 3 } \cline { 5 - 6 } E1 & Gas/flow rate [sccm $]$ & Time $[\mathrm{min}]$ & & Gas/flow rate [sccm] & Time [min] \\
E2 & $\mathrm{Ar} / 400 \mathrm{O}_{2} / 100$ & 5 & & $\mathrm{Ar} / 500$ & 5 \\
E3 & $\mathrm{Ar} / 450 \mathrm{O}_{2} / 50$ & 5 & & $\mathrm{Ar} / 500$ & 5 \\
E4 & $\mathrm{O}_{2} / 500$ & 5 & & $\mathrm{Ar} / 500$ & 5 \\
E5 & $\mathrm{Ar} / 400 \mathrm{O}_{2} / 100$ & 5 & & $\mathrm{Ar} / 500$ & 7 \\
E6 & $\mathrm{Ar} / 400 \mathrm{O}_{2} / 100$ & 5 & & $\mathrm{Ar} / 500$ & 10 \\
\hline
\end{tabular}

The sheet resistivity and transmittance of ITO films on DPSS are shown in Fig. 2. The transmittance was measured after the annealing procedures (one-step or two-step). Results presented in Fig. 2(a) indicate that $\mathrm{S} 1$ obtains a reduced sheet resistivity of around $2 \times 10^{-4} \Omega \mathrm{cm}$ after the first annealing in the Ar atmosphere. Meanwhile, the transmittance of S1 after the first annealing (presented in Fig. 2(b)) was greater than $96 \%$ in the $590 \mathrm{~nm}$ to $780 \mathrm{~nm}$ wavelength range. However, if $\mathrm{S} 1$ carried out additional annealing in the $\mathrm{Ar} / \mathrm{O}_{2}$ mixture atmosphere, the sheet resistivity in Fig. 2(a) would become worse than that of the as-deposited one, despite better transmittance shown in Fig. 2(b). This implies that the single annealing in the Ar atmosphere can improve the performance of ITO layers, but the following annealing in the $\mathrm{Ar} / \mathrm{O}_{2}$ mixture atmosphere is not the right way to obtain better sheet resistivity and transmittance.

Next, the opposite annealing procedure was explored. Based on the results of S2 and S3, although the sheet resistivity becomes worse after the first annealing in the $\mathrm{Ar} / \mathrm{O}_{2}$ mixture atmosphere, the second annealing in the Ar atmosphere can reduce it to the same level of S1 after the single annealing in the Ar atmosphere. These results indicated that the sheet resistivity of ITO largely depended on the final annealing atmosphere. If the final annealing was in the Ar atmosphere, the sheet resistivity could obtain a lower value; but if the final annealing was in the $\mathrm{O}_{2}$-containing atmosphere, the sheet resistivity would obtain a higher value.

Generally, the sheet resistivity of ITO is related to the grain boundary scattering (average grain size) and oxygen vacancies [35]. The average grain sizes of ITO, obtained from XRD diagrams in Fig. 3 by Debye-Scherer's formula [33], were reduced after the second annealing in the Ar atmosphere (Table 3). This reduction implied that the grain boundary scattering after the second annealing in the Ar atmosphere was slightly enhanced, which would decrease the carrier mobility and increase the sheet resistivity. Therefore, the grain boundary scattering didn't contribute to the reduction of the sheet resistivity of ITO after the second Ar annealing.

Instead, we attributed this reduction to oxygen vacancies. Figures 3(c) and 3(d) show the XPS spectra of O-1s peak of sputtered ITO after annealing in different atmospheres. By using Gaussian functions, we determined two peaks located at around $529.8 \mathrm{eV}\left(\mathrm{O}_{\text {I }}\right.$ peak) and $531.2 \mathrm{eV}$ 


\section{Optics EXPRESS}
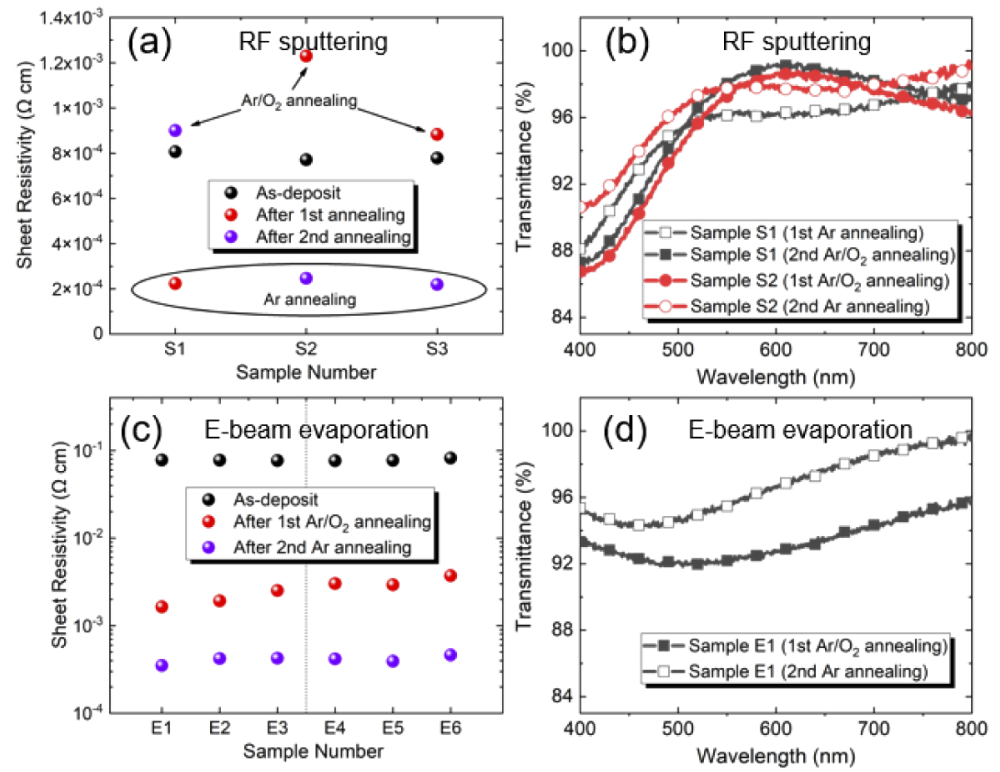

Fig. 2. (a) The sheet resistivity and (b) transmittance of ITO layers deposited by RF sputtering under different annealing processes. (c) The sheet resistivity and (d) transmittance of ITO layers deposited by e-beam evaporation under different annealing processes.
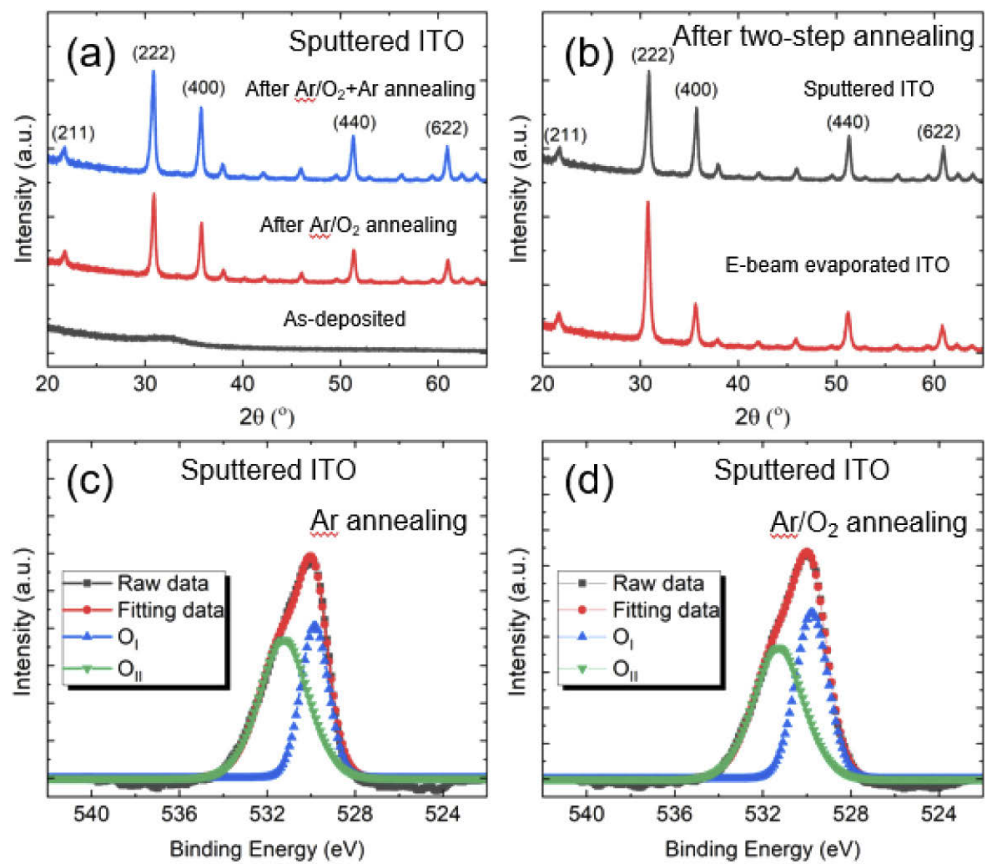

Fig. 3. XRD diagrams of (a) 111-nm-thick sputtered ITO and (b) its comparison with 114-nm-thick e-beam evaporated ITO after two-step annealing. XPS spectra of the O-1s peak from the sputtered ITO after the thermal annealing in the (a) Ar atmosphere and (b) $\mathrm{Ar} / \mathrm{O}_{2}$ atmosphere 
Table 3. FWHM of (222) and (400) peaks for sputtered ITO and e-beam evaporated ITO.

\begin{tabular}{ccccccc}
\hline \multirow{2}{*}{ Annealing } & \multicolumn{2}{c}{$\mathrm{FWHM} / 2 \theta\left(^{\circ} /{ }^{\circ}\right)$} & & \multicolumn{2}{c}{ Average grain size $(\mathrm{nm})$} \\
\cline { 3 - 4 } \cline { 6 - 7 } Sputtered ITO & $\mathrm{Ar} / \mathrm{O}_{2}$ & $0.385^{\circ} / 30.89^{\circ}$ & $0.366^{\circ} / 35.78^{\circ}$ & & 23.8 & \\
& $\mathrm{Ar} / \mathrm{O}_{2}+\mathrm{Ar}$ & $0.424^{\circ} / 30.85^{\circ}$ & $0.410^{\circ} / 35.72^{\circ}$ & & 21.6 & 25.3 \\
e-beam evaporated ITO & $\mathrm{Ar} / \mathrm{O}_{2}+\mathrm{Ar}$ & $0.479^{\circ} / 30.76^{\circ}$ & $0.478^{\circ} / 35.62^{\circ}$ & & 19.1 & 22.6 \\
\hline
\end{tabular}

$\left(\mathrm{O}_{\text {II }}\right.$ peak), which came from In-O and oxygen vacancies $\left(\mathrm{In}-\mathrm{V}_{\mathrm{o}}\right)$ [28]. The integrated area ratio of the two peaks $\left(\mathrm{O}_{\mathrm{II}} / \mathrm{O}_{\mathrm{I}}\right)$ for sputtered ITO annealed in the Ar atmosphere was larger than that for sputtered ITO annealed in the $\mathrm{Ar} / \mathrm{O}_{2}$ atmosphere (Table 4). This larger ratio confirmed that additional oxygen vacancies were created after the annealing in the Ar atmosphere, leading to the higher carrier concentration and lower sheet resistivity [35].

Table 4. Fitting results of $0-1 \mathrm{~s}$ peaks of sputtered ITO after annealing in different atmospheres.

\begin{tabular}{cccc}
\hline & $\mathrm{O}_{\text {I }}$ peak $(\mathrm{eV})$ & $\mathrm{O}_{\text {II }}$ peak $(\mathrm{eV})$ & Integrated area ratio $\left(\mathrm{O}_{\text {II }} / \mathrm{O}_{\mathrm{I}}\right)$ \\
\hline Ar annealing & 529.84 & 531.24 & 1.59 \\
$\mathrm{Ar} / \mathrm{O}_{2}$ annealing & 529.78 & 531.29 & 1.20 \\
\hline
\end{tabular}

Meanwhile, compared to S1 after the single annealing in the Ar atmosphere, the transmittance of S2 after the two-step annealing, presented in Fig. 2(b), is slightly increased (by 1-2\%) in the $590 \mathrm{~nm}$ to $780 \mathrm{~nm}$ wavelength range. This means the two-step annealing under $\mathrm{O}_{2}$-containing and $\mathrm{O}_{2}$-poor atmospheres in the sequence can obtain both good sheet resistivity and transmittance, and is demonstrated as the better annealing procedure. However, we found the transmittance would have an acceptable sacrifice of within $1 \%$ in the $590 \mathrm{~nm}$ to $700 \mathrm{~nm}$ wavelength range after the second annealing in the Ar atmosphere, when comparing the first and second annealing of S2 that is presented in Fig. 2(b).

In the case of ITO by e-beam evaporation, the annealing processes were designed in the same sequence. For the first annealing process in an $\mathrm{O}_{2}$-containing atmosphere, the influence of the different $\mathrm{Ar} / \mathrm{O}_{2}$ ratios (Samples E1-E3) was investigated under a constant total flow rate. As shown on the left side of Fig. 2(c), E1 obtains the lowest sheet resistivity of around $3.5 \times 10^{-4}$ $\Omega \mathrm{cm}$ after the second Ar annealing. Because the second annealing uses the same conditions, the atmosphere of the first annealing was optimized as the $\mathrm{Ar} / \mathrm{O}_{2}(400 \mathrm{sccm} / 100 \mathrm{sccm})$. Figure 2(d) shows that the second annealing in the Ar atmosphere can further enhance the transmittance of E1 to above $95 \%$ in the $590 \mathrm{~nm}$ to $780 \mathrm{~nm}$ wavelength range, instead of having a sacrifice like sputtered ITO. Finally, the annealing time of the second Ar process was optimized based on the samples E1 and E4-E6. We found that E1 exhibits the minimum value of sheet resistivity, which is shown in Fig. 2(c). Meanwhile, the transmittance of E1 was quite similar to the others with a different second annealing time (not shown here), demonstrating the best time for the second annealing is 5 minutes. Based on all the results discussed above, we conclude that no matter the ITO layers that are deposited by RF sputtering or e-beam evaporation, the two-step annealing process under $\mathrm{O}_{2}$-containing and $\mathrm{O}_{2}$-poor atmosphere in the sequence helps to achieve excellent performances in both sheet resistivity and transmittance, which are regarded as critical factors for transparent conductive layers. It is worth noting that the second annealing in the $\mathrm{Ar}$ atmosphere not only improves the performances, but also makes the results reproducible, which is vital for production.

In addition, the thickness of ITO layers by RF sputtering was optimized. The results of sheet resistivity and transmittance are shown in Figs. 4(a) and 4(b), respectively. The sheet resistivity of sputtered ITO was found to be independent of thickness, always as low as $1.6 \times 10^{-4} \Omega \mathrm{cm}$. Meanwhile, the transmittances of the samples with thicknesses of $148 \mathrm{~nm}$ or less maintained over 


\section{Optics EXPRESS}

$98 \%$ in the $590 \mathrm{~nm}$ to $780 \mathrm{~nm}$ wavelength range, which was higher than that of the sample with a $185 \mathrm{~nm}$ thickness. Therefore, this suggests that the optimal thickness of ITO should be less than $148 \mathrm{~nm}$ for the highest transmittance.
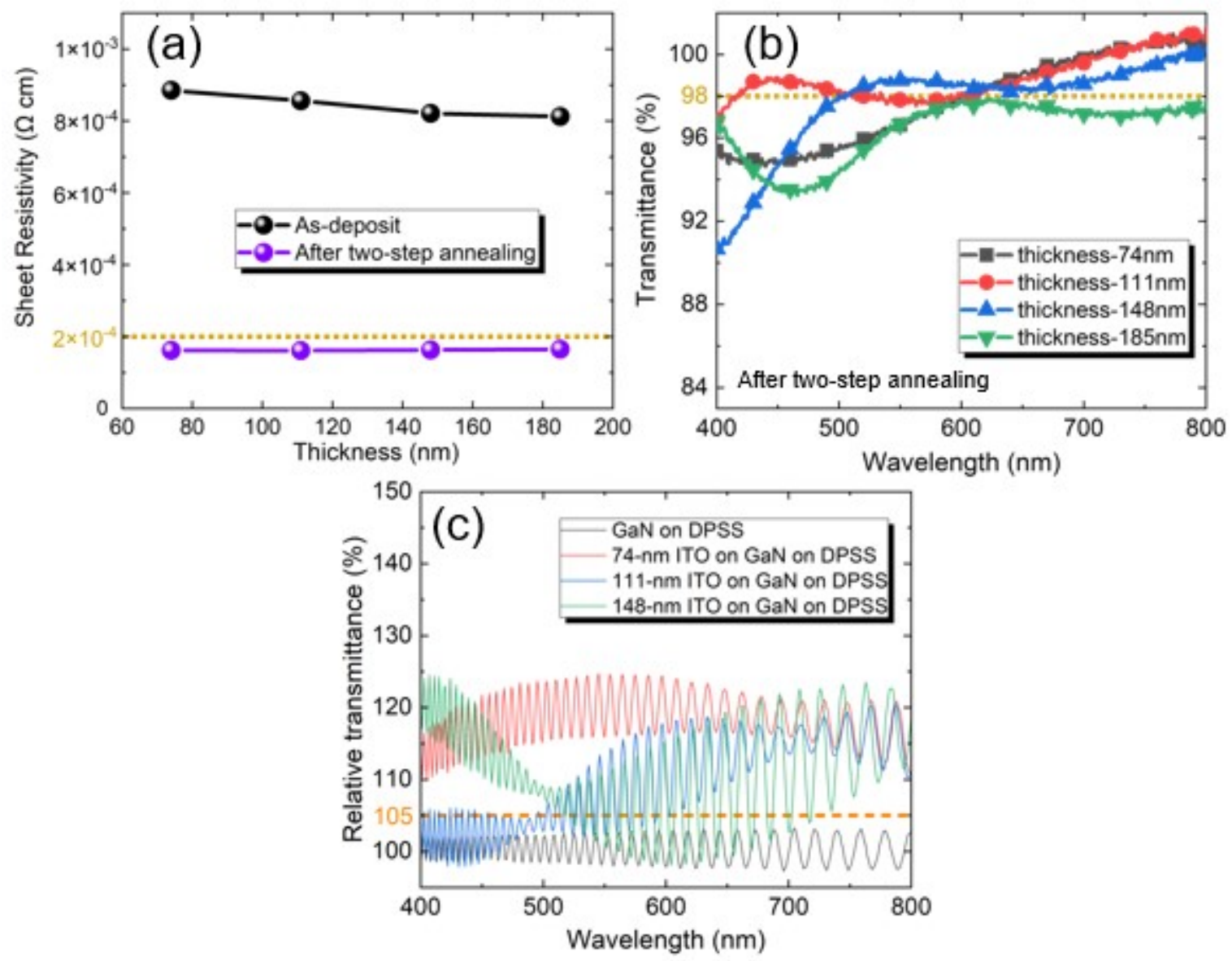

Fig. 4. (a) The sheet resistivity and (b) transmittance of sputtered ITO with different thicknesses on DPSS. (c) The relative transmittance of sputtered ITO with different thicknesses on GaN on DPSS.

Moreover, we found that sputtered ITO exhibited better performances in terms of both conductivity and transmittance when compared to ITO with the same thickness deposited by e-beam evaporation. The better performance of sputtered ITO was attributed to the better crystallinity. From the XRD data in Fig. 3(b), we obtained the FWHM and $2 \theta$ of (222) and (400) peaks for sputtered ITO and e-beam evaporated ITO, as shown in Table 3. The calculated averaged grain sizes of sputtered ITO was larger than those of e-beam evaporated ITO, leading to the improved conductivity of sputtered ITO. Meanwhile, the better crystallinity indicated that less deep energy levels in sputtered ITO, which resulted in less absorption and enhanced the optical transmittance. Therefore, sputtered ITO was superior to that deposited by e-beam evaporation in terms of conductivity and optical transmittance.

In the LED device structures, ITO was deposited on GaN instead of sapphire substrates. The thickness of ITO would influence the light extraction of LEDs [36]. Therefore, we deposited ITO by sputtering on GaN on DPSS and measured the relative transmittance, as shown in Fig. 4(c). The relative transmittance of GaN on DPSS oscillated around $100 \%$ because we used similar 


\section{Optics EXPRESS}

GaN on DPSS as the reference sample. And for ITO with different thicknesses on GaN on DPSS, the relative transmittances were all above $105 \%$ when we considered average oscillation centers of interference fringes. Besides, 74-nm-thick ITO on GaN exhibited the highest transmittance of $115-120 \%$ in the amber and red region. Thus, considering the light extraction of amber and red LEDs, we determined that the optimal thickness of sputtered ITO should be around $74 \mathrm{~nm}$.

Finally, ITO layers deposited by RF sputtering and e-beam evaporation were utilized for amber and red LEDs. The total thickness of ITO by sputtering and e-beam evaporation was $69 \mathrm{~nm}$ and $80 \mathrm{~nm}$, respectively. To avoid the damage of the p-GaN surface by sputtering [37], we introduced the first ITO layer $(15 \mathrm{~nm})$ by low-power and high-pressure sputtering on the p-GaN layer. This additional sputtered ITO layer was demonstrated to reduce the forward voltages at $20 \mathrm{~mA}$ for blue LEDs fabricated from commercial epitaxial wafers compared to single sputtered ITO layer. Therefore, we adopted these two sputtered ITO layers for p-contacts in amber and red LEDs. Figures 5(a) and 5(b) show the current-voltage (I-V) curves of amber and red LEDs, respectively. The typical behavior of the $I-V$ curves indicates that both amber and red LEDs have good electrical performance. The averaged forward voltages at $20 \mathrm{~mA}$ are $3.34 \mathrm{~V}$ and $3.36 \mathrm{~V}$ for the amber and red LEDs, respectively, with sputtered ITO layers (see Fig. 5(c)). They are lower than those of devices with ITO layers by e-beam evaporation. It is worth noting that the forward voltages of our amber and red LEDs are much better than previously reported ones (> 4 V) $[10,21]$.
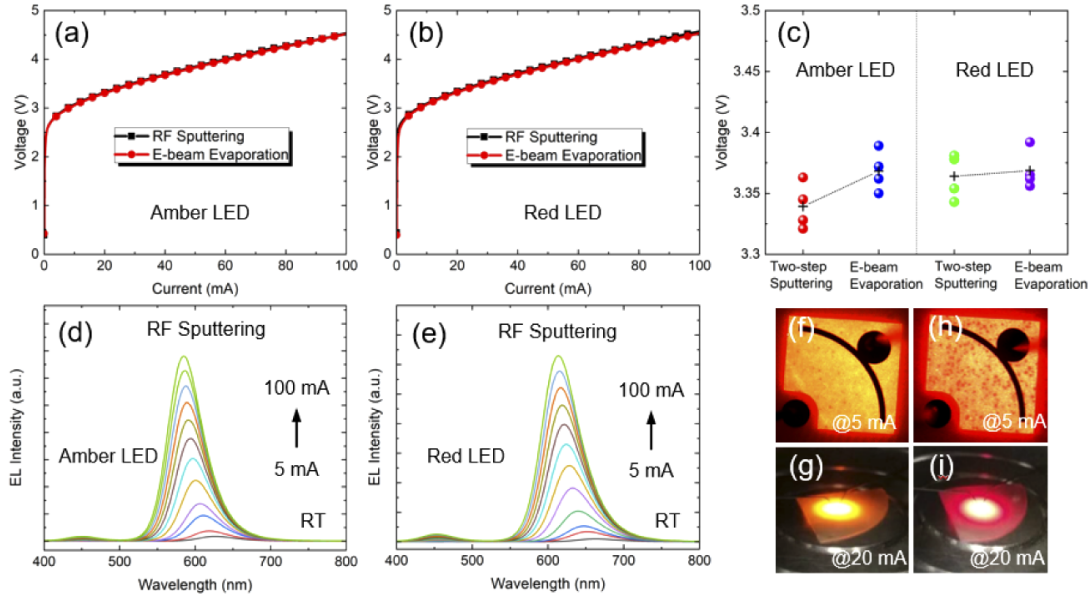

Fig. 5. (a) \& (b) The $I-V$ curves of InGaN-based amber and red LEDs with ITO layers by RF sputtering and e-beam evaporation, respectively. (c) The forward voltages during $20 \mathrm{~mA}$ current injection. The cross symbols are the average values from four samples with e-beam evaporation and RF sputtering, respectively. (d) \& (e) The EL spectra of InGaN-based amber and red LEDs with sputtered ITO after current injections ranging from $5 \mathrm{~mA}$ to $100 \mathrm{~mA}$, respectively. A picture of one chip and a bird view of (f) \& (g) amber and (h) \& (i) red LEDs.

The RT EL spectra were measured on the wafer during injections of $5 \mathrm{~mA}$ to $100 \mathrm{~mA}$ currents (Figs. 5(d) and 5(e)). The emission wavelength of amber and red LEDs is around $600 \mathrm{~nm}$ and $640 \mathrm{~nm}$ during $20 \mathrm{~mA}$ current injection, respectively. However, both amber and red LEDs have an additional peak emission at the $450 \mathrm{~nm}$ wavelength, which is believed to originate from the phase-separated component in the high-In-content InGaN double QWs [13,14]. The device emission images of amber and red LEDs at $5 \mathrm{~mA}$ and $20 \mathrm{~mA}$ are shown in Figs. 5(f)-(i), respectively. Compared to the amber LED, the red LED continues to have many dark spots, which 


\section{Optics EXPRESS}

might be related to threading dislocations or trench defects and needs to be further improved in crystal quality.

The light output power-current $(L-I)$ characteristics of amber and red LEDs are shown in Figs. 6(a) and 6(b), respectively. We measured the relative light output power of each wafer using identical optical set-ups. The results from amber and red LEDs with sputtered ITO layers were found to have $15.6 \%$ and $13.0 \%$ improvements at $20 \mathrm{~mA}$, respectively, compared to those with ITO by e-beam evaporation. Because sputtered ITO with a thickness of $69 \mathrm{~nm}$ and $80 \mathrm{~nm}$ should have similar electrical and optical performances, we attributed the better device performance to the improved electrical and optical properties of sputtered ITO layers compared to those of ITO by e-beam evaporation.
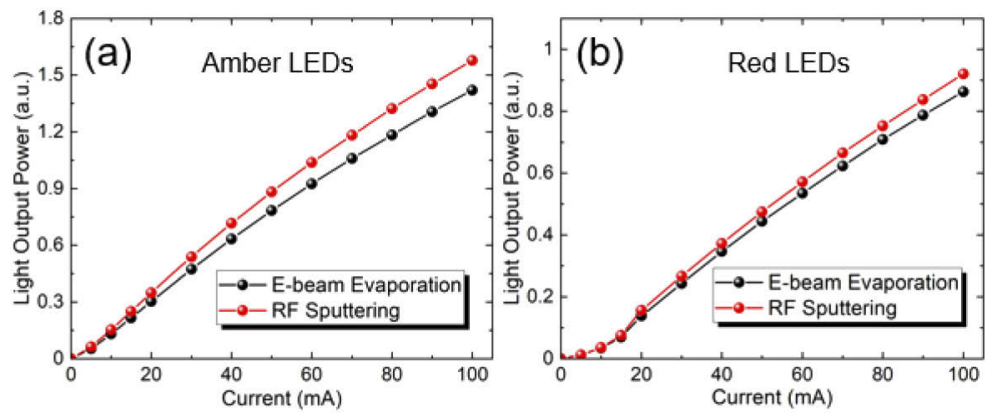

Fig. 6. The $L-I$ characteristics of (a) amber and (b) red LEDs with different ITO deposition methods.

\section{Conclusion}

In summary, the characteristics of ITO deposited by RF sputtering and e-beam evaporation were manipulated to better serve as good transparent conductive layers. The ITO layers that underwent a two-step annealing process were found to have low sheet resistivity $\left(1.6 \times 10^{-4} \Omega \mathrm{cm}\right)$ and high transmittance (more than 98\%) in the amber and red spectrum range. Also, double ITO layers by RF sputtering were found to make excellent contact with the $\mathrm{p}-\mathrm{GaN}$ layer, obtaining a reduced forward voltage at $20 \mathrm{~mA}$. The application of optimal double ITO layers on InGaN-based amber and red LEDs enhanced light output power by $15.6 \%$ and $13.0 \%$, respectively, compared to those using ITO layers by e-beam evaporation.

\section{Funding}

King Abdullah University of Science and Technology (BAS/1/1676-01-01).

\section{Acknowledgements}

This work was supported by King Abdullah University of Science and Technology (KAUST).

\section{Disclosures}

The authors declare no conflicts of interest.

\section{References}

1. H. Amano, N. Sawaki, I. Akasaki, and Y. Toyoda, "Metalorganic vapor phase epitaxial growth of a high quality GaN film using an AlN buffer layer," Appl. Phys. Lett. 48(5), 353-355 (1986).

2. S. Nakamura, "GaN Growth Using GaN Buffer Layer," Jpn. J. Appl. Phys. 30(Part 2, No. 10A), L1705-L1707 (1991).

3. H. Amano, M. Kito, K. Hiramatsu, and I. Akasaki, "P-Type Conduction in Mg-Doped GaN Treated with Low-Energy Electron Beam Irradiation (LEEBI),” Jpn. J. Appl. Phys. 28(Part 2, No. 12), L2112-L2114 (1989). 


\section{Optics EXPRESS}

4. S. Nakamura, T. Mukai, M. Senoh, and N. Iwasa, "Thermal Annealing Effects on P-Type Mg-Doped GaN Films," Jpn. J. Appl. Phys. 31(Part 2, No. 2B), L139-L142 (1992).

5. S. Nakamura, M. Senoh, and T. Mukai, "High-power InGaN/GaN double-heterostructure violet light emitting diodes," Appl. Phys. Lett. 62(19), 2390-2392 (1993).

6. Y. Narukawa, M. Ichikawa, D. Sanga, M. Sano, and T. Mukai, "White light emitting diodes with super-high luminous efficacy," J. Phys. D: Appl. Phys. 43(35), 354002 (2010).

7. B. Damilano and B. Gil, "Yellow-red emission from (Ga, In)N heterostructures," J. Phys. D: Appl. Phys. 48(40), 403001 (2015).

8. A. Vaitkevičius, J. Mickevičius, D. Dobrovolskas, Ö Tuna, C. Giesen, M. Heuken, and G. Tamulaitis, "Influence of quantum-confined Stark effect on optical properties within trench defects in InGaN quantum wells with different indium content," J. Appl. Phys. 115(21), 213512 (2014).

9. T. Mukai, M. Yamada, and S. Nakamura, "Characteristics of InGaN-Based UV/Blue/Green/Amber/Red Light-Emitting Diodes,” Jpn. J. Appl. Phys. 38(Part 1, No. 7A), 3976-3981 (1999).

10. J.-I. Hwang, R. Hashimoto, S. Saito, and S. Nunoue, "Development of InGaN-based red LED grown on (0001) polar surface," Appl. Phys. Express 7(7), 071003 (2014).

11. K. Ohkawa, T. Watanabe, M. Sakamoto, A. Hirako, and M. Deura, "740-nm emission from InGaN-based LEDs on c-plane sapphire substrates by MOVPE," J. Cryst. Growth 343(1), 13-16 (2012).

12. K. Ohkawa, F. Ichinohe, T. Watanabe, K. Nakamura, and D. Iida, "Metalorganic vapor-phase epitaxial growth simulation to realize high-quality and high-In-content InGaN alloys," J. Cryst. Growth 512, 69-73 (2019).

13. D. Iida, S. Lu, S. Hirahara, K. Niwa, S. Kamiyama, and K. Ohkawa, "Enhanced light output power of InGaN-based amber LEDs by strain-compensating AlN/AlGaN barriers," J. Cryst. Growth 448, 105-108 (2016).

14. D. Iida, K. Niwa, S. Kamiyama, and K. Ohkawa, "Demonstration of InGaN-based orange LEDs with hybrid multiple-quantum-wells structure," Appl. Phys. Express 9(11), 111003 (2016).

15. D. Iida, Z. Zhuang, P. Kirilenko, M. Velazquez-Rizo, and K. Ohkawa are submitting a manuscript entitled " $633-\mathrm{nm}$ InGaN-based red LEDs grown on thick GaN underlying layers with reducing in-plane residual stress".Appl. Phys. Lett.To be published.

16. D. Iida, Z. Zhuang, P. Kirilenko, M. Velazquez-Rizo, and K. Ohkawa, "Demonstration of low forward voltage InGaN-based red LEDs," Appl. Phys. Express 13(3), 031001 (2020).

17. W. Lv, L. Wang, J. Wang, Y. Xing, J. Zheng, D. Yang, Z. Hao, and Y. Luo, "Green and Red Light-Emitting Diodes Based on Multilayer InGaN/GaN Dots Grown by Growth Interruption Method,” Jpn. J. Appl. Phys. 52(8S), 08JG13 (2013).

18. I. L. Koslow, M. T. Hardy, P. S. Hsu, P.-Y. Dang, F. Wu, A. Romanov, Y.-R. Wu, E. C. Young, S. Nakamura, J. S. Speck, and S. P. DenBaars, "Performance and polarization effects in (1122) long wavelength light emitting diodes grown on stress relaxed InGaN buffer layers," Appl. Phys. Lett. 101(12), 121106 (2012).

19. M. R. Philip, D. D. Choudhary, M. Djavid, K. Q. Le, J. Piao, and H. P. T. Nguyen, "High efficiency green/yellow and red InGaN/AlGaN nanowire light-emitting diodes grown by molecular beam epitaxy," J. Sci.: Adv. Mater. Devices 2(2), 150-155 (2017).

20. T. Ozaki, M. Funato, and Y. Kawakami, "Red-emitting $\mathrm{In}_{x} \mathrm{Ga}_{1-\mathrm{x}} \mathrm{N} / \mathrm{In}_{\mathrm{y}} \mathrm{Ga}_{1-\mathrm{y}} \mathrm{N}$ quantum wells grown on lattice-matched $\mathrm{In}_{\mathrm{y}} \mathrm{Ga}_{1-\mathrm{y}} \mathrm{N} / \mathrm{ScAlMgO}$ (0001) templates," Appl. Phys. Express 12(1), 011007 (2019).

21. B. Mitchell, V. Dierolf, T. Gregorkiewicz, and Y. Fujiwara, "Perspective: Toward efficient GaN-based red light emitting diodes using europium doping," J. Appl. Phys. 123(16), 160901 (2018).

22. S. Figge, R. Kröger, T. Böttcher, P. L. Ryder, and D. Hommel, "Magnesium segregation and the formation of pyramidal defects in p-GaN," Appl. Phys. Lett. 81(25), 4748-4750 (2002).

23. L. T. Romano, M. Kneissl, J. E. Northrup, C. G. V. D. Walle, and D. W. Treat, "Influence of microstructure on the carrier concentration of Mg-doped GaN films," Appl. Phys. Lett. 79(17), 2734-2736 (2001).

24. D. L. Hibbard, S. P. Jung, C. Wang, D. Ullery, Y. S. Zhao, H. P. Lee, W. So, and H. Liu, "Low resistance high reflectance contacts to p-GaN using oxidized Ni/Au and Al or Ag," Appl. Phys. Lett. 83(2), 311-313 (2003).

25. J.-S. Jang and T.-Y. Seong, "Low-resistance and thermally stable indium tin oxide Ohmic contacts on strained p-In ${ }_{0.15} \mathrm{Ga}_{0.85} \mathrm{~N} / \mathrm{p}-\mathrm{GaN}$ layer," J. Appl. Phys. 101(1), 013711 (2007).

26. J. L. Chen and W. D. Brewer, "Ohmic Contacts on p-GaN," Adv. Electron. Mater. 1(8), 1500113 (2015).

27. H. L. Hao, L. K. Wu, W. J. Chung, Y. Zhang, and W. Z. Shen, "Process optimization of RTA on the characteristics of ITO-coated GaN-based LEDs," Microelectron. Reliab. 55(11), 2263-2268 (2015).

28. A. Chen, K. Zhu, H. Zhong, Q. Shao, and G. Ge, "A new investigation of oxygen flow influence on ITO thin films by magnetron sputtering," Sol. Energy Mater. Sol. Cells 120, 157-162 (2014).

29. M. Marikkannan, M. Subramanian, J. Mayandi, M. Tanemura, V. Vishnukanthan, and J. M. Pearce, "Effect of ambient combinations of argon, oxygen, and hydrogen on the properties of DC magnetron sputtered indium tin oxide films," AIP Adv. 5(1), 017128 (2015).

30. H. M. Lo, S. C. Shei, X. F. Zeng, S. Chang, and H. Lin, "AlGaInP-Based LEDs With a $\mathrm{p}^{+}-$GaP Window Layer and a Thermally Annealed ITO Contact," IEEE J. Quantum Electron. 47(6), 803-809 (2011).

31. S. L. Ou, D. S. Wuu, S. P. Liu, Y. C. Fu, S. C. Huang, and R. H. Horng, "Pulsed laser deposition of ITO/AZO transparent contact layers for GaN LED applications," Opt. Express 19(17), 16244-16251 (2011). 


\section{Optics EXPRESS}

32. M. M. Aliyu, S. Hossain, J. Husna, N. Dhar, M. Q. Huda, K. Sopian, and N. Amin, "High quality indium tin oxide (ITO) film growth by controlling pressure in RF magnetron sputtering," in 2012 38th IEEE Photovoltaic Specialists Conference (IEEE, 2012), pp. 002009-002013.

33. K.-K. Kim, H. Kim, S.-N. Lee, and S. Cho, "Structural, optical, and electrical properties of E-beam and sputterdeposited ITO films for LED applications," Electron. Mater. Lett. 7(2), 145-149 (2011).

34. S. Song, T. Yang, J. Liu, Y. Xin, Y. Li, and S. Han, "Rapid thermal annealing of ITO films," Appl. Surf. Sci. 257(16), 7061-7064 (2011).

35. C. Guillén and J. Herrero, "Structure, optical, and electrical properties of indium tin oxide thin films prepared by sputtering at room temperature and annealed in air or nitrogen," J. Appl. Phys. 101(7), 073514 (2007).

36. W. Hou, C. Stark, S. You, L. Zhao, T. Detchprohm, and C. Wetzel, "Evaluation of metal/indium-tin-oxide for transparent low-resistance contacts to p-type GaN," Appl. Opt. 51(23), 5596-5600 (2012).

37. K. J. Son, T. K. Kim, Y.-J. Cha, S. K. Oh, S.-J. You, J.-H. Ryou, and J. S. Kwak, "Impact of Plasma Electron Flux on Plasma Damage-Free Sputtering of Ultrathin Tin-Doped Indium Oxide Contact Layer on p-GaN for InGaN/GaN Light-Emitting Diodes,” Adv. Sci. 5(2), 1700637 (2018). 